\title{
Swarm Optimization Methods in Microwave Imaging
}

\author{
Andrea Randazzo \\ Department of Naval, Electrical, Electronic, and Telecommunication Engineering, University of Genoa, Via Opera Pia 11A, \\ 16145 Genova, Italy
}

Correspondence should be addressed to Andrea Randazzo, randazzo@dibe.unige.it

Received 8 August 2012; Accepted 21 September 2012

Academic Editor: Kristen M. Donnell

Copyright ( $) 2012$ Andrea Randazzo. This is an open access article distributed under the Creative Commons Attribution License, which permits unrestricted use, distribution, and reproduction in any medium, provided the original work is properly cited.

Swarm intelligence denotes a class of new stochastic algorithms inspired by the collective social behavior of natural entities (e.g., birds, ants, etc.). Such approaches have been proven to be quite effective in several applicative fields, ranging from intelligent routing to image processing. In the last years, they have also been successfully applied in electromagnetics, especially for antenna synthesis, component design, and microwave imaging. In this paper, the application of swarm optimization methods to microwave imaging is discussed, and some recent imaging approaches based on such methods are critically reviewed.

\section{Introduction}

Microwave imaging denotes a class of noninvasive techniques for the retrieval of information about unknown conducting/dielectric objects starting from samples of the electromagnetic field they scatter when illuminated by one or more external microwave sources [1]. Such techniques have been acquiring an ever growing interest thanks to their ability of directly retrieving the distributions of the dielectric properties of targets in a safe way (i.e., with nonionizing radiation) and with quite inexpensive apparatuses. In recent years, several works concerned those systems. In particular, their ability to provide excellent diagnostic capabilities has been assessed in several areas, including civil and industrial engineering [2], nondestructive testing and evaluation (NDT\&E) [3], geophysical prospecting [4], and biomedical engineering [5].

The development of effective reconstruction procedures is, however, still a quite difficult task. The main difficulties are related to the underlying mathematical problem. In fact, the information about the target are contained in a complex way inside the scattered electric field. In particular, the governing equations turn out to be highly nonlinear and strongly ill posed. Consequently, inversion procedures are usually quite complex and time consuming, especially when high resolution images are needed.
In the literature several approaches have been proposed for solving this problem. In particular, two main classes of algorithms can be identified. Deterministic [6-21] and stochastic strategies [22-33]. Deterministic methods are usually fast and, when converge, they produce high quality reconstructions. However, their main drawback is that they are local approaches, that is, they usually require to be started with an initial guess "near" enough to the correct solution. Otherwise, such approaches can be trapped in local minima corresponding to false solutions. Moreover, in most cases, it is difficult to introduce a priori information in the reconstruction process. On the contrary, stochastic approaches are global optimization methods, that is, they are able to find the global solution of the problem. Furthermore, thanks to their flexibility, they usually easily allow the introduction of a priori information on the unknowns. The main drawback of this class of approaches is their computational burden. However, it should be noted that with the recent growth of computational resources, it can be envisioned that future generation computers will allow faster reconstructions.

Stochastic approaches are usually based on a population of trial solutions that is iteratively updated. Depending on how the population is modified at each iteration, different class of methods can be identified. The "classical" approaches 
have been developed in order to simulate the evolutionary processes of biological entities. Such kind of methods are now very common in several areas of electromagnetic engineering and, in particular, in nondestructive testing and imaging. Among them, the most successful ones are the genetic algorithm (GA) [34] and the differential evolution (DE) method [35].

A new class of stochastic approaches has been recently introduced by mimicking the collective behavior of real entities such as particles, birds, and ants. Such approaches, usually referred as swarm methods [36, 37], have been proven to be quite effective in several applications, where they outperform standard evolutionary methods. Recently, they are becoming very popular in electromagnetics, too. Several applications, ranging from antenna synthesis to microwave component design, have been proposed in the literature. Moreover, several different approaches, such as the particle swarm optimization (PSO) $[38,39]$ and the ant colony algorithm (ACO) [40, 41], have been successfully applied to microwave imaging.

In the present paper, the application of swarm algorithms to microwave imaging is discussed, and some of the recent literature results are critically reviewed. The paper is organized as follows. In Section 2, the mathematical framework of optimization problems for microwave imaging is briefly recalled. Section 3 describes the considered swarm methods. Section 4 reviews the applications of such algorithms in the framework of microwave imaging. Finally, conclusions are drawn in Section 5.

\section{Microwave Imaging as an Optimization Problem}

Microwave imaging approaches aim at retrieving information about unknown objects (e.g., the full distribution of dielectric properties, the shape in the case of conducting targets, the position and size of an inclusion, etc.) starting from measures of the electromagnetic field, they scatter when illuminated by a known incident electric field.

Despite different equations are needed for modeling different problems (e.g., two-dimensional or three-dimensional problems, dielectric or perfectly conducting objects, and so on), it is usually possible to write a relationship between the desired unknown quantities and the measured field (at least in implicit form), that is, an equation of the form

$$
F(x)=e,
$$

where $x$ is the unknown function describing the searched features of the object (e.g., $x=\epsilon_{r}$ when dealing with the reconstruction of the distribution of the dielectric permittivity), and $e$ is the measured (vector or scalar) electric field, that is, the known data of the equation. Consequently, the inverse problem can be recast as an optimization problem by defining a cost function of the form

$$
f(x)=w\|e-F(x)\|_{2}^{2},
$$

where $w$ is a constant normalization parameter. In (2), the standard Euclidean 2-norm has been considered. This is often a common choice in microwave imaging and allows the use of widely studied mathematical tools for the analysis of the convergence and regularization behaviors. However, different norms have been recently proposed, too (e.g., the norm of $L^{p}$ Banach spaces [42]).

For illustrative purposes, the case of cylindrical dielectric targets embedded in free space is explicitly described in the following. The cylinder axis is assumed to be parallel to the $z$ axis. A time-harmonic (with angular frequency $\omega$ ) transverse magnetic (TM) incident field is assumed. Similar expressions can be derived for other configurations (e.g., half-space and multilayer media, three-dimensional vector problems, etc.).

When dealing with inhomogeneous dielectric objects embedded in an infinite and homogeneous medium, the electromagnetic inverse scattering problem is governed by the following two operator equations [1]:

$$
\begin{gathered}
e_{\text {scatt }}(\mathbf{r})=G_{\text {ext }}\left(c e_{\text {tot }}\right)(\mathbf{r}), \quad \mathbf{r} \in D_{\text {obs }}, \\
e_{\text {inc }}(\mathbf{r})=e_{\text {tot }}(\mathbf{r})-G_{\text {int }}\left(c e_{\text {tot }}\right)(\mathbf{r}), \quad \mathbf{r} \in D_{\text {inv }},
\end{gathered}
$$

where $G_{\text {ext }}(\cdot)(\mathbf{r})=-k^{2} \int_{D_{\text {inv }}}(\cdot) g_{0}\left(\mathbf{r}, \mathbf{r}^{\prime}\right) d \mathbf{r}^{\prime}, \mathbf{r} \in D_{\text {obs }}$ (being $D_{\text {obs }}$ the observation domain where the scattered electric field is collected), and $G_{\text {int }}(\cdot)(\mathbf{r})=-k^{2} \int_{D_{\text {inv }}}(\cdot) g_{0}\left(\mathbf{r}, \mathbf{r}^{\prime}\right) d \mathbf{r}^{\prime}$, $\mathbf{r} \in D_{\text {inv }}$ (being $D_{\text {inv }}$ the investigation area where the target is located), are data and state operators whose kernel is the free-space Green's function $g_{0}$ (being $k=\omega \sqrt{\epsilon_{0} \mu_{0}}$ the freespace wavenumber), $c(\mathbf{r})=\epsilon_{r}(\mathbf{r})-1$ is the contrast function (being $\epsilon_{r}$ the space dependent relative complex dielectric permittivity of the investigation area $\left.D_{\text {inv }}\right), e_{\text {inc }}$ and $e_{\text {tot }}$ are the $z$-components of the incident and total electric fields inside the investigation area, and $e_{\text {scatt }}$ is the $z$-component of the scattered electric field in the points of the observation domain $D_{\text {obs }}[1]$.

In discrete setting, the two equations in (3) can be replaced by the following matrix equations:

$$
\begin{gathered}
\mathbf{e}_{\text {scatt }}=\mathbf{G}_{\text {ext }} \operatorname{diag}(\mathbf{c}) \mathbf{e}_{\mathrm{tot}}, \\
\mathbf{e}_{\text {inc }}=\mathbf{e}_{\mathrm{tot}}-\mathbf{G}_{\mathrm{int}} \operatorname{diag}(\mathbf{c}) \mathbf{e}_{\mathrm{tot}},
\end{gathered}
$$

where $\mathbf{c}$ is an array containing the $N$ unknown coefficients of the expansion of $c$ in a given set of basis functions, $\mathbf{e}_{\text {inc }}$ and $\mathbf{e}_{\text {tot }}$ are arrays of dimensions $N$ containing the coefficients of the incident and total electric fields, and $\mathbf{e}_{\text {scatt }}$ is an array of $M$ elements containing the coefficients used to represent the known scattered field in the measurement domain.

As in the continuous case, the equations in (4) represent an ill-conditioned nonlinear problem. Directly solving this problem is very difficult. However, as previously introduced, it is possible to recast its solution as the minimization of a proper cost function. Usually, the following functional is considered

$$
\begin{aligned}
f(\mathbf{x})= & w_{D}\left\|\mathbf{e}_{\text {scatt }}-\mathbf{G}_{\text {ext }} \operatorname{diag}(\mathbf{c}) \mathbf{e}_{\text {tot }}\right\|_{2}^{2} \\
& +w_{S}\left\|\mathbf{e}_{\text {inc }}-\mathbf{e}_{\text {tot }}+\mathbf{G}_{\text {int }} \operatorname{diag}(\mathbf{c}) \mathbf{e}_{\text {tot }}\right\|_{2}^{2},
\end{aligned}
$$

where $w_{D}$ and $w_{S}$ are weighting parameters, often chosen equal to $w_{D}=\left\|\mathbf{e}_{\text {scatt }}\right\|_{2}^{-2}$ and $w_{S}=\left\|\mathbf{e}_{\text {inc }}\right\|_{2}^{-2}$. In this case, the unknown array $\mathbf{x}$ is composed by the elements of $\mathbf{c}$ and $\mathbf{e}_{\text {tot }}$. 
Clearly, other forms can be used, too. For example, the two equations can be combined together in order to obtain the following cost function:

$$
f(\mathbf{x})=w_{D}\left\|\mathbf{e}_{\text {scatt }}-\mathbf{G}_{\text {ext }} \operatorname{diag}(\mathbf{c})\left(\mathbf{I}-\mathbf{G}_{\text {int }} \operatorname{diag}(\mathbf{c})\right)^{-1} \mathbf{e}_{\text {inc }}\right\|_{2}^{2},
$$

which has the advantage of considering only the contrast function coefficients as unknowns. As a drawback, it needs a matrix inversion, leading to a high computational cost.

The cost function can be modified by introducing multiview and multifrequency information. In this case, the target is illuminated by several incident fields (e.g., generated by $S$ sources located all around the objects and operating at $F$ different frequencies), and the cost function becomes

$$
\begin{aligned}
f(\mathbf{x})= & w_{D} \sum_{f=1}^{F} \sum_{s=1}^{S}\left\|\mathbf{e}_{\mathrm{scatt}}^{f, s}-\mathbf{G}_{\mathrm{ext}}^{f, s} \operatorname{diag}(\mathbf{c}) \mathbf{e}_{\mathrm{tot}}^{f, s}\right\|_{2}^{2} \\
& +w_{S} \sum_{f=1}^{F} \sum_{s=1}^{S}\left\|\mathbf{e}_{\mathrm{inc}}^{f, s}-\mathbf{e}_{\mathrm{tot}}^{f, s}+\mathbf{G}_{\mathrm{int}}^{f, s} \operatorname{diag}(\mathbf{c}) \mathbf{e}_{\mathrm{tot}}^{f, s}\right\|_{2}^{2},
\end{aligned}
$$

where the indexes $f$ and $s$ denote that the corresponding quantities are related to the $f$ th operating frequency and the sth illumination.

\section{Swarm Optimization Algorithms}

Swarm algorithms belong to the class of optimization methods, that is, they find the minimum of a given cost function $f(x)$. Similarly to other evolutionary approaches, they usually allow reaching the global optimum, thus avoiding to find a suboptimal solution corresponding to a local minima. Moreover, they are able to easily incorporate constraints on the search space. However, while evolutionary algorithms are inspired by the genetic adaptation of organisms, swarm methods exploit their collective social behavior.

In order to define a general framework for swarm methods, let us consider a cost function $f: S \subseteq \mathbb{R}^{G} \rightarrow$ $[0,+\infty)$ to be minimized (or maximized). For sake of simplicity, the case of bound constraints (i.e., $l_{g} \leq x_{g} \leq u_{g}$, being $l_{g}$ and $u_{g}$ the lower and upper bounds on the $g$ th component of $\mathbf{x}$ ) is considered in the following. However, the unknown array $\mathbf{x}=\left[x_{1}, x_{2}, \ldots, x_{G}\right]^{t} \in S \subseteq \mathbb{R}^{G}$ can be subjected to arbitrary constraints.

Swarm algorithms are usually iterative methods based on a population of $P$ trial solutions $\wp_{k}=\left\{\mathbf{x}_{p}^{(k)}, p=1, \ldots\right.$, $P$ \} (being $k$ the iteration number) representing $P$ agents inspired from real world (e.g., particles, birds, ants, etc.). The population is iteratively modified according to rules aimed at mimicking the natural behavior of those agents.

In the framework of electromagnetic imaging, the following swarm algorithms have been mainly considered.

(i) Particle swarm optimization.

(ii) Ant colony optimization.

(iii) Artificial bee colony optimization.
Apart from the standard approaches, hybridization with other methods (e.g., local optimization methods, other evolutionary approaches, machine learning algorithms, etc.) has also been proposed in the literature.

In the following sections, some information about the basic versions of those methods are briefly recalled.

3.1. Particle Swarm Optimization (PSO). PSO is inspired by the behavior of flocks of birds and shoals of fish [38, 39]. Each entity moves through the space of solutions with a velocity that is related to the locations and cost function values of the members of the swarm. In particular, the basic PSO algorithm [38] considers a set of $p=1, \ldots, P$ "particles" characterized, at each iteration $k$, by their positions $\mathbf{x}_{p}^{(k)}$ and velocity $\mathbf{v}_{p}^{(k)}$. If no a priori information is available, usually the algorithm is initialized by using random values, that is,

$$
\begin{aligned}
& x_{p, g}^{(0)}=l_{g}+\left(u_{g}-l_{g}\right) U(0,1), \\
& v_{p, g}^{(0)}=l_{g}^{v}+\left(u_{g}^{v}-l_{g}^{v}\right) U(0,1), \\
& g=1, \ldots, G, \quad p=1, \ldots, P,
\end{aligned}
$$

where $l_{g}$ and $u_{g}$ are the lower and upper bounds for the $g$ th component of particles' positions, $l_{g}^{v}$ and $u_{g}^{v}$ are the lower and upper bounds for the gth component of the particles' velocities, and $U(0,1)$ is a function returning a random variable uniformly distributed between 0 and 1 . Clearly, if some a priori information is available, the initialization scheme can be modified for taking it into account (e.g., in microwave imaging, when the aim is the identification of one or more localized objects, it is possible to generate random targets and use them as starting random solutions).

The trial solutions are iteratively updated by using the following two-step scheme (as also shown in the flow chart in Figure 1):

$$
\begin{gathered}
\mathbf{v}_{p}^{(k+1)}=\omega^{(k)} \mathbf{v}_{p}^{(k)}+\eta_{1} U(0,1)\left(\mathbf{p}_{p}-\mathbf{x}_{p}^{(k)}\right) \\
+\eta_{2} U(0,1)\left(\mathbf{g}-\mathbf{x}_{p}^{(k)}\right), \\
\mathbf{x}_{p}^{(k+1)}=\mathbf{x}_{p}^{(k)}+\mathbf{v}_{p}^{(k)},
\end{gathered}
$$

where $\omega^{(k)}$ is the inertia parameter, $\eta_{1}$ and $\eta_{2}$ are acceleration coefficients, and $\mathbf{p}_{p}$ and $\mathbf{g}$ are the best solution achieved by the $p$ th particle and by the whole swarm so far, respectively. The two acceleration terms in the velocity update can be thought as two elastic forces with random magnitude attracting the particles to the best solutions achieved so far by each entity and by the whole swarm, respectively. After the new solutions are generated the values of $\mathbf{p}_{p}$ and $\mathbf{g}$ are updated.

The procedure is iterated until some predefined stopping criteria is fulfilled. In particular, the stopping criteria can be composed by several conditions. Some of the most commonly used are the following.

(i) Maximum number of iterations: the method is stopped when a given number of iteration $k_{\max }$ is reached. 


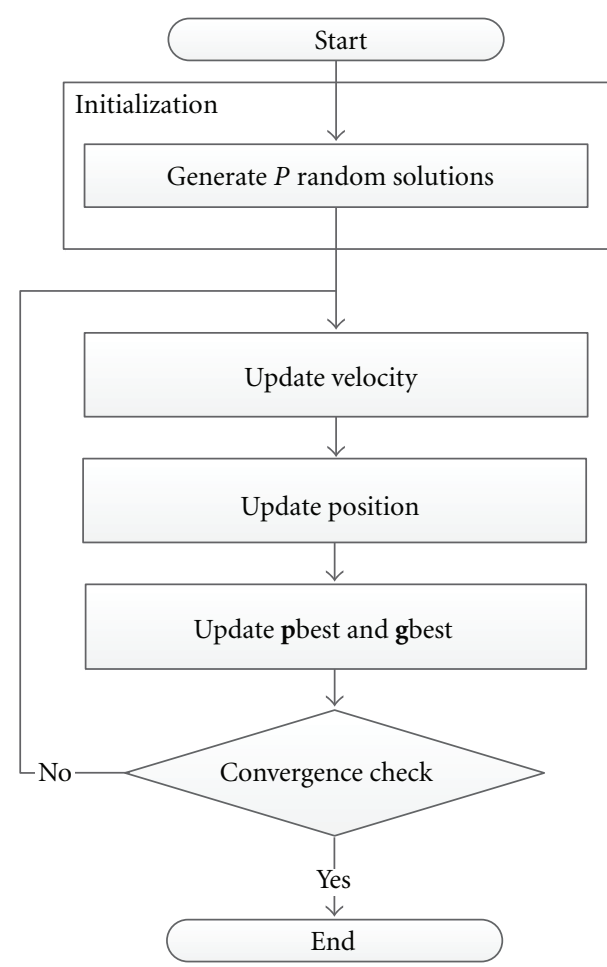

Figure 1: Flow chart of the PSO algorithm.

(ii) Cost function threshold: the method is stopped when the value of cost function of the best trial solution falls below a given threshold $f_{\text {th }}$.

(iii) Cost function improvement threshold: the method is stopped if the improvement of the cost function of the best individual after $k_{\text {th }}$ iterations is below a fixed threshold $\Delta f_{\text {th }}$.

3.2. Ant Colony Optimization. Ant colony optimization (ACO) is a recently developed swarm optimization method based on the behavior of ants, and, in particular, on how they find the optimal path for reaching the food starting from their nest [40]. Initially, ants explore the area around their nest in a random manner searching for food. When a food source is found, ants evaluate it and bring back some food to the nest depositing a pheromone trail on the ground during the trip. The amount of pheromone depends on the quantity and quality of the food, and it is used to guide other ants to the food source. It has been found that the pheromone trails allow ants to find the shortest path between nest and food sources. On the basis of such behavior, ACO was initially designed for solving hard combinatorial problems, such as the traveling salesman problem [41]. Successively, the original algorithm has been extended for considering different applications. In particular, several efforts have been devoted to the extension to continuous domains, and some different versions of the algorithm have been proposed [43-45]. In the following, the $\mathrm{ACO}_{\mathbb{R}}$ version [46] is described. Such algorithm can be considered as composed by three functional blocks, as shown in Figure 2: initialization, solution construction, and

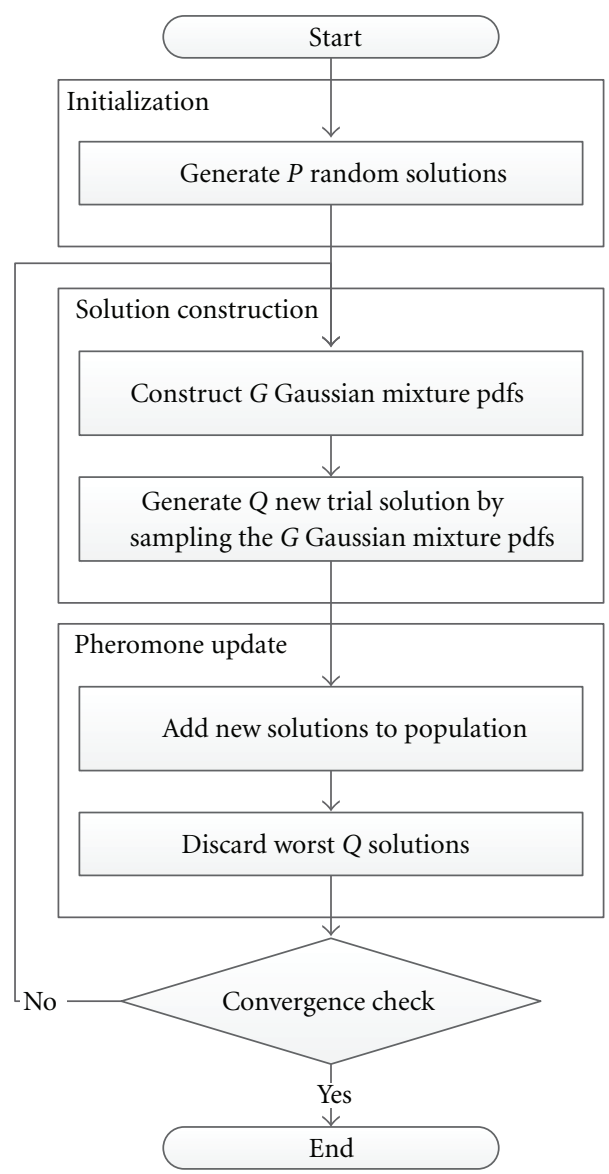

FIgUre 2: Flow chart of the $\mathrm{ACO}_{\mathbb{R}}$ algorithm.

pheromone update. The algorithm iterates until a predefined stopping criteria is satisfied.

In the initialization block, the initial population $\wp_{0}=$ $\left\{\mathbf{x}_{p}^{(0)}, p=1, \ldots, P\right\}$ is created by generating $P$ random trial solutions $\mathbf{x}_{p}^{(0)}=\left[x_{p, 1}^{(0)}, \ldots, x_{p, G}^{(0)}\right]^{t}$. Similarly to the PSO algorithm, when no a priori information is available and assuming boundary constraints, the $g$ th components of the $p$ th trial solution are generated by sampling a uniform distribution as defined in (8). In this case, too, if additional a priori information is available, it can be included in the initialization procedure.

The solution construction block is used to generate new trial solutions. In particular, at the $k$ th iteration, $Q$ new solutions $\widetilde{\mathbf{x}}_{q}^{(k)}, q=1, \ldots, Q$ are generated by sampling a set of Gaussian mixture probability density functions. In particular, the probability density function of the $g$ th component is built as

$$
G_{g}^{(k)}(x)=\sum_{p=1}^{P} w_{p} \frac{1}{s_{p, g}^{(k)} \sqrt{2 \pi}} e^{-\left(x-m_{p, g}^{(k)}\right)^{2} / 2\left(s_{k, p, g}^{(k)}\right)^{2}},
$$

where the weighting parameters $w_{p}, p=1, \ldots, P$, are given by

$$
w_{p}=\frac{1}{\rho P \sqrt{2 \pi}} e^{-(p-1)^{2} / 2 \rho^{2} P^{2}}, \quad p=1, \ldots, P,
$$


and the mean, $m_{p, g}^{(k)}$, and standard deviation, $s_{p, g}^{(k)}$, of the Gaussian kernels are given by

$$
\begin{gathered}
m_{p, g}^{(k)}=x_{p, g}^{(k)}, \quad p=1, \ldots, P, g=1, \ldots, G, \\
s_{p, g}^{(k)}=\xi \sum_{i=1}^{P} \frac{\left|x_{p, g}^{(k)}-x_{i, g}^{(k)}\right|}{P-1}, \quad p=1, \ldots, P, g=1, \ldots, G .
\end{gathered}
$$

In the previous equations, $\rho$ is the pheromone evaporation rate, and $\xi$ is a scaling parameter. Such quantities are key parameters of the ACO algorithm [46], and the best choice for their values depends on the specific application.

The pheromone update block is responsible of the population update. In particular, two mechanism are used to build the new population $\wp_{k+1}$ of the $(k+1)$ th iteration:

(i) positive update: a temporary population is created by adding newly created solutions to the solution archive. A pool of $P+Q$ trial solutions $\tilde{\wp}_{k+1}=$ $\left\{x_{k, p}, p=1, \ldots, P\right\} \bigcup\left\{\tilde{x}_{k, q}, q=1, \ldots, Q\right\}$ is then obtained,

(ii) negative update: the worst $Q$ elements of $\tilde{\wp}_{k+1}$ (i.e., those characterized by the higher values of the cost function) are discarded. The remaining $P$ solutions constitute the new population $\wp_{k+1}$.

In order to speed up this stage, usually the solution archive is ordered on the basis of the cost function, that is, $f\left(x_{k, 1}\right) \leq f\left(x_{k, 2}\right) \leq \cdots \leq f\left(x_{k, P}\right)$. The two previous blocks are iteratively applied since some predefined stopping criteria is fulfilled. In this case, too, the stopping criteria can be a combination of several different conditions.

3.3. Artificial Bee Colony Optimization. The artificial bee colony algorithm is a swarm optimization method introduced by Karaboga [47] and Karaboga and Basturk [48] and inspired by the foraging behavior of honey bees. In particular, $A B C$ is based on the model proposed in [49], which defines two main self-organizing and collective intelligence behaviors: recruitment of foragers for working on rich food sources and abandonment of poor sources. In $\mathrm{ABC}$, a colony of artificial bees search for rich artificial food sources (representing solutions of the considered optimization problem) by iteratively employing the following two strategies: movement towards better solutions by means of a neighbor search mechanism and abandonment of poor solutions that cannot be further improved.

In the following, an artificial colony, composed by $C$ entities, is considered. Bees can be classified in three groups: employed (i.e., already working on a known food source), onlooker (i.e., waiting for a food source), and scout (i.e., randomly searching for new sources) bees. The trial solutions are the food sources associated with the employed bees. Let us denote by $P$ the number of employed bees (corresponding to the number of trial solutions). Often, such number is set equal to $P=C / 2$, and the number of onlooker bees is chosen equal to $P$. A block diagram of the method is shown in Figure 3.

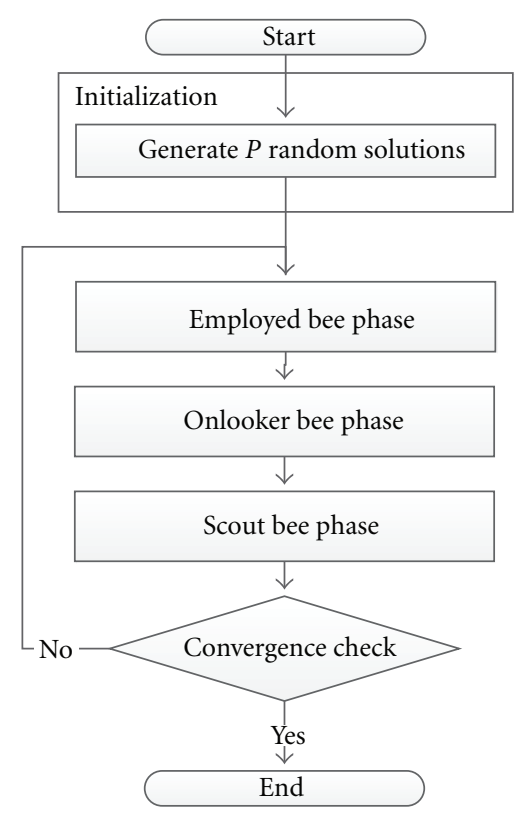

Figure 3: Flow chart of the $A B C$ algorithm.

In the initialization phase, the $P$ trial solution are randomly generated, for example, by using a relationship similar to that in (8). The cost function of all trial solutions is evaluated and stored. In the employed bee phase, the employed bees modify their trial solutions according to

$$
x_{p, g}^{(k+1)}=x_{p, g}^{(k)}+U(0,1)\left(x_{p, g}^{(k)}-x_{h, g}^{(k)}\right),
$$

where $g$ and $h$ are randomly chosen.

In the onlooker bee phase, each onlooker bee select a food source in a probabilistic way. In particular, the probability of choosing the $p$ th food source is given by

$$
p_{p}=\frac{f_{\text {fit }}\left(\mathbf{x}_{p}^{(k)}\right)}{\sum_{p=1}^{P} f_{\text {fit }}\left(\mathbf{x}_{p}^{(k)}\right)},
$$

where $f_{\text {fit }}$ is a fitness function defined as $f_{\text {fit }}(\mathbf{x})=1 /(f(\mathbf{x})+1)$. The food sources selected by the onlooker bees are further improved by using (13).

If a food source is not improved after a predefined number of iterations $K_{\lim }$ (food source limit), that is, $f\left(\mathbf{x}_{p}^{(k)}\right) \geq f\left(\mathbf{x}_{p}^{(k-q)}\right)$ for $q=1, \ldots, K_{\lim }$, it is abandoned by its employed bee. Such bee becomes a scout bee and starts searching for a new food source randomly. Consequently, in the scout bee phase, employed bees working on abandoned sources generate new trial solution in a random way (i.e., by using the same relationship used in the initialization phase). After a new solution is found, the scout bees are reverted to employed bees, and they start working on the new food sources. Clearly, at the beginning of the optimization process, no scout bees are present and consequently, for the first iterations, only the solutions initially discovered in the initialization phase are processed. 
TABLE 1: Overview of swarm algorithms applications in microwave imaging.

\begin{tabular}{|c|c|c|c|c|}
\hline Object type & Material & Illumination type & Method & Reference \\
\hline 1D profile & Dielectric & Time domain & PSO & {$[50-52]$} \\
\hline 2D cylinders & Dielectric & Time domain & PSO & {$[53,54]$} \\
\hline 2D cylinders & Dielectric & Single frequency & PSO & {$[55-58]$} \\
\hline 2D cylinders & PEC & Single frequency & PSO & {$[59,60]$} \\
\hline 3D objects & Dielectric & Single frequency & PSO & {$[58,61]$} \\
\hline 3D objects & Dielectric & Single frequency & $\mu \mathrm{PSO}$ & {$[62]$} \\
\hline 2D cylinders & Dielectric & Time domain & APSO & {$[63]$} \\
\hline 2D cylinders & PEC & Time domain & APSO & {$[64,65]$} \\
\hline 2D cylinders & PEC & Singe frequency & PSO-SA & {$[66]$} \\
\hline 2D cylinders & Dielectric & Single frequency & HSPO & {$[67]$} \\
\hline 2D cylinders & Dielectric & Single frequency & PSO-RBF & {$[68]$} \\
\hline 2D cylinders & Dielectric & Single frequency & IMSA-PSO & {$[69-73]$} \\
\hline 3D objects & Dielectric & Single frequency & IMSA-PSO & {$[74,75]$} \\
\hline 2D cylinders & Dielectric & Single frequency & $\mathrm{ACO}$ & {$[28,76]$} \\
\hline 2D cylinders & Dielectric & Single frequency & ACO-LSM & [77] \\
\hline 3D objects & Dielectric & Single frequency & ACO-LSM & {$[78,79]$} \\
\hline 3D objects & Dielectric & Time domain & $\mathrm{ABC}$ & {$[80]$} \\
\hline 2D cylinders & Dielectric & Single frequency & $\mathrm{ABC}$ & This paper \\
\hline
\end{tabular}

\section{Application of Swarm Optimization to Microwave Imaging}

Swarm algorithms have been used for solving different types of microwave imaging problems. In particular, twodimensional and three-dimensional dielectric and PEC targets have been considered in the literature. Moreover, both single-frequency, multifrequency, and time-domain incident radiation has been used. An overview of papers proposing swarm optimization methods in microwave imaging is given in Table 1. Specific details are provided in the following sections.

4.1. PSO. PSO has been extensively used in electromagnetic problems [39]. Several works proposed PSO, also with enhancement to the standard algorithm, for microwave imaging problems. In $[50,51]$, the reconstruction of onedimensional dielectric profiles, illuminated by a Gaussian pulse (plane waves are assumed), is considered. Both noiseless and noisy data have been used. Two size of the populations are considered equal and twice the number of unknowns. The acceleration coefficients of the PSO are $\eta_{1}=$ $\eta_{2}=0.5$, and the inertia parameters is initially set to 1 and decreased linearly to 0.7 in 500 iterations (maximum number of iterations). A comparison with the $\mathrm{DE}$ is also provided. In the reported numerical results, the PSO shows slightly better convergence rate, but the DE allows obtaining a more precise reconstruction. A similar approach is proposed in [52], too.

In [53], the authors propose the use of the PSO for the localization of dielectric circular cylinders under a TM timedomain formulation. The unknowns are the position, size, and dielectric properties of the target. Good reconstructions are obtained by using five particles.
In [54], the reconstruction of homogeneous dielectric cylinders is considered. The external shape of the cylinder is described by using a spline representation. The unknowns are the parameters of the spline and the dielectric permittivity. The proposed approach is able to reconstruct such quantities with errors less than about 7\% (shape) and 3\% (permittivity) for signal-to-noise ratio above $10 \mathrm{~dB}$.

In [55], the reconstruction of the distribution of the relative dielectric permittivity of $2 \mathrm{D}$ dielectric structures is concerned. A single-frequency multiview TM illumination is considered. The cost function defined in (7) is used. A population size of twice the unknowns number is used; the acceleration coefficients are randomly generated in the range $[0,2]$, and the inertia parameter is set equal to 0.4 . The reported results show that the PSO is able to provide good reconstruction of the considered objects. Moreover, comparisons with a genetic algorithm and with the conjugate gradient (CG) method are provided. For the considered case, PSO outperforms both GA and CG (the mean relative reconstruction errors are $1.7 \%, 1.8 \%$, and 5.7\%, resp.). A similar approach is proposed in [56], too, providing similar conclusions. In [57], the PSO is used to find the parameters of a crack in the outer layer of a two-layer dielectric cylinder. The direct solver is based on a finite difference frequency Domain (FDFD) scheme. Good agreements are obtained for several positions of the cracks. The same approach is also applied in [58] to the detection of a tumor inside a model of breast. Both 2D and 3D simplified configurations are assumed. The unknowns are the position and shape of the malignant inclusion.

The reconstruction of the shape of PEC cylinders is considered in $[59,60]$. A cubic-spline-based representation is used for defining the shape of the cylinder. The target 
is illuminated by TM waves impinging from 7 directions uniformly distributed around it and, for each illumination, the scattered field is collected in 32 points. Single-frequency operation is assumed. The maximum radius is approximately $1.5 \lambda$ (being $\lambda$ the wavelength in the background medium). Under such assumptions, good reconstructions have been obtained by considering 10 control points for the splines, $\eta_{1}=\eta_{2}=0.5$, and population sizes ranging from 10 to 60 . The inertia is initialized to 1.0 and decreased to 0.7 after 200 iterations (maximum number of iterations). A comparison with DE is also performed. Both algorithms provide good results, although DE, for the considered cases, usually produces lower values of the cost function and of the reconstruction error.

In [61], 3D objects are reconstructed. The measurement configuration simulates those employed for breast cancer detection, that is, four circular arrays of 36 antennas are located around a cubic investigation domain. In this reference, the PSO-based approach is able to retrieve a small centered inclusions.

In [62], a $\mu \mathrm{PSO}$ algorithm is proposed for tackling the high dimensionality of the microwave imaging problems. Satisfactory reconstructions are obtained in the reconstruction of a 3D model of breast with a malignant inclusion by using only 5 particles. Comparisons with the standard PSO (with swarm size of 25 particles) show that the new approach is able to obtain comparable results, but with a smaller population size.

In [63], an asynchronous PSO (APSO) is proposed for the reconstruction of the location, shape, and permittivity of a dielectric cylinder illuminated by TM pulses. The direct solver is based on a finite difference time-domain (FDTD) scheme. The main difference with respect to the standard PSO is the population updating mechanism. In the APSO, the new best position is computed after every particle update, and it is used in the following updates immediately. Consequently, the swarm reacts more quickly. The same approach is used in $[64,65]$ for the identification of the shape of PEC cylinders in free space and inside a dielectric slab. In such cases, the APSO is able to correctly identify the shape of the targets with an error of about 5\% (in presence of noise on the data) and it shows better performance with respect to standard PSO.

Some hybrid versions of the PSO have also been proposed in the literature. In [66], PSO is combined with simulated annealing (SA) for exploiting the exploration properties of PSO and the exploitation ability of SA. The reported results concern the reconstruction of the external shape of a cylinder under multiview TM illumination and show that the hybrid approach allows reaching better results than the standard one (reconstructions errors were 0.0014 and 0.072, resp.). In [67], a hybrid PSO (HPSO) is used for reconstructing dielectric cylinders under TM illuminations. The difference from the standard PSO is mainly related to the use of a particle swarm crossover for enhancing trial solutions. In [68], the PSO is combined with radial basis function (RBF) networks. In particular, the RBF is used to obtain an estimate of the dielectric properties of two-dimensional cylinders under single-frequency TM illumination. The PSO is employed to efficiently training the RBF starting from a set of simulated configurations.

In [69-71], an integrated multiscaling approach (IMSA) relying upon PSO is presented. In such technique, the investigation area is iteratively reconstructed at different scales. At every scale, the PSO is used to obtain a quantitative reconstruction of the distribution of the dielectric properties. A clustering techniques is used to identify the scatterers, and then the investigation area is refined in order to focus only on the objects. The proposed approach is tested by using several different two-dimensional targets illuminated by monochromatic TM incident waves. The reported results confirm that the integrated strategy is able to outperform its standard counterpart. Moreover, the new approach is also able to provide better results than those obtained by using the CG and GA (both in their standard form and inserted in a IMSA framework). As an example, a square hollow cylinder $\left(\epsilon_{r}=1.5\right.$, sides $L_{\text {in }}=0.8 \lambda$ and $\left.L_{\text {out }}=1.6 \lambda\right)$ contained in an investigation area of side $2.4 \lambda$ and illuminated by plane waves impinging from 4 different directions (with the electric field measured in 21 points located on a circumference of radius $1.8 \lambda$ for every view) is efficiently reconstructed in 4 steps. At each step, the PSO is executed with 20 particles, $\eta_{1}=\eta_{2}=2.0, \omega^{(k)}=0.4$, and $k_{\max }=2000$ (the mesh used to discretized the investigation area has size 36 ). The obtained reconstruction error is $3.8 \%$. For the same configuration, the multiscaling version of CG provides an error of $4.6 \%$. An experimental validation with the Fresnel data [81] is also provided, confirming the capabilities of the approach when working in real environments. In $[72,73]$, the IMSA-PSO is tested on phaseless data (i.e., amplitude-only scattered field measures are available). In this case, too, good agreement with the actual profile are obtained. In $[74,75]$, the IMSAPSO is also extended to the reconstruction of $3 \mathrm{D}$ objects.

4.2. Ant Colony Optimization-Based Imaging Algorithms. ACO has been used in several electromagnetic applications, in particular for design of antennas and microwave components [82, 83], for the allocation of base stations [84], and for microwave imaging. Concerning electromagnetic imaging, ACO has been applied both to the reconstruction of $2 \mathrm{D}$ cylindrical structures and 3D objects.

In [76], the ACO algorithm is applied to the reconstruction of multiple dielectric lossless cylinders under TM illumination. A two-dimensional formulation is assumed. Two representations of the dielectric properties are considered, pixelbased and splinebased. The number of unknowns, in the two cases, are 256 and 169 , respectively. The population size is set equal to the number of unknowns and, at every iteration, $Q=P / 10$ new solutions are created. The parameters of the ACO are set equal to $\rho=0.1$ and $\xi=0.85$ (according to the suggestions available in the literature). The iterations are stopped when a maximum number of iterations, $k_{\max }=2000$, is reached. The provided results show that in all cases the ACO-based approach is able to correctly reconstruct the targets with a mean relative error lower than 5\%. Moreover, as expected, the spline representation allows for a faster convergence (thanks to the lower number of unknowns). 
In [28], ACO is applied to a similar configuration (twodimensional imaging of a two-layer dielectric cylinders). A comparison with GA and DE is provided. The reconstruction results show that in the considered case ACO "reaches a more accurate reconstruction than those obtained by the other two methods" and "requires a lower number of function evaluations."

In [77], a hybrid method is proposed for two-dimensional imaging of multiple homogeneous dielectric targets. First, the shape of the targets is estimated by using the linear sampling method (LSM), which is a fast and efficient qualitative approach able to retrieve the support of the scatterers starting from the scattered field data. After this step, the ACO is applied to retrieve the values of the relative dielectric permittivity and the electric conductivity. In this way, the ACO only needs to find a few parameters (two for every objects identified by the LSM). The approach has been extended to three-dimensional targets in $[78,79]$, where the full vector problem with arbitrary homogeneous dielectric targets is taken into account. Single and multiple dielectric objects are reconstructed with good accuracy and with low computational efforts. As an example, for a rectangular parallelepiped of dimensions $0.66 \lambda \times 0.33 \lambda \times 0.5 \lambda$, after the support estimation, mean relative reconstruction errors equal to $2.5 \%$ (dielectric permittivity) and $7 \%$ (electric conductivity) are obtained with 45.6 cost function evaluations (mean value).

4.3. Artificial Bee Colony-Based Imaging Algorithms. The $\mathrm{ABC}$ has been applied for breast cancer detection in [80]. A full three-dimensional configuration is considered. The breast is modeled both by using simplified structures and a realistic MRI-based phantom. ABC is employed for the reconstruction of the position, size, and dielectric properties of the malignant inclusion (supposed of spherical size and homogeneous). The problem size is thus $G=6$. A population of $P=10$ bees is employed. In the reported test cases, $\mathrm{ABC}$ is able to reach the convergence in less than 30 iteration. Moreover, the algorithm is able to estimate the position and size of the tumor with satisfactory accuracy (e.g., in the simplified case, the localization error is less than $10 \%$ and the size estimation error is less than $1 \mathrm{~mm}$ ). A comparison with PSO, DE, and GA is also provided. In the considered case, ABC outperforms the other approaches (GA provided the worst results PSO and DE gave similar results).

An example of use of the $\mathrm{ABC}$ for the reconstruction of the full distribution of the dielectric properties of unknown objects is reported in the following. Cylindrical scatterers under TM illumination are considered. A multiview configuration is assumed, that is, $S=8$ line-current sources uniformly spaced on a circumference of radius $1.5 \lambda$ are sequentially used for illuminating the objects. The scattered electric field is collected in 51 points uniformly spaced on an angular sector of 270 degrees on the same circumference (positioned such that the source lies in the sector without measurement probes). The investigation domain is a square area of side $2 \lambda$. The input data (scattered electric field) are computed by using a numerical code based on the method of moments [85] with pulse basis and Dirac's delta

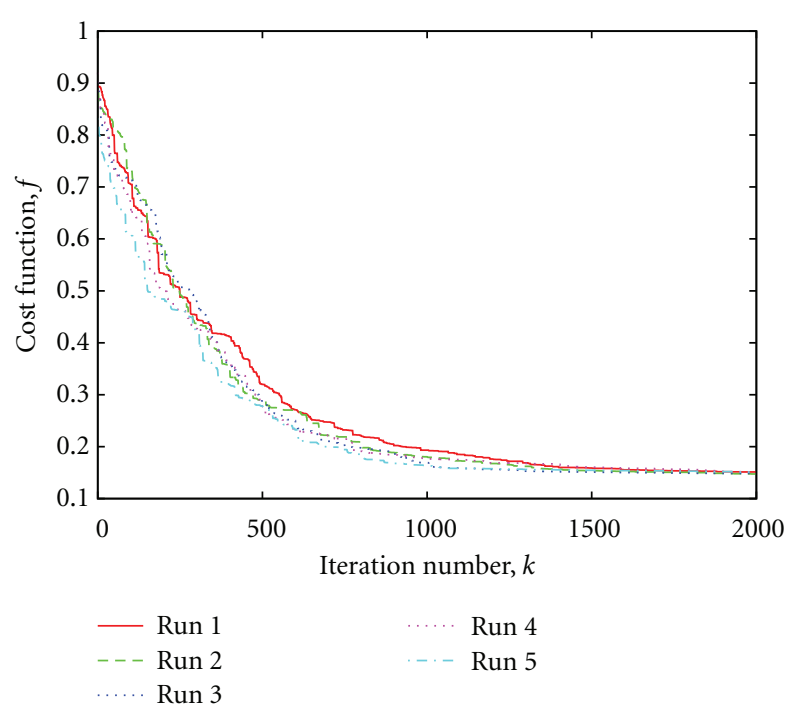

FIGURE 4: Cost function versus the iteration number. ABC-based inversion algorithm.

weighting functions. A finer mesh is used for solving the forward problem in order to avoid inverse crimes. Moreover, the computed electric field is corrupted with a Gaussian noise with zero mean value and variance corresponding to a signal-to-noise ratio of $25 \mathrm{~dB}$. In the inversion procedure, the investigation area is discretized into $N=256$ square subdomains, and the unknowns are the values of the relative dielectric permittivity in such cells. Two separate targets are located in the investigation area: a circular cylinder (radius $0.25 \lambda$, center $(-0.25 \lambda, 0.25 \lambda)$, relative dielectric permittivity 2.0 ) and a square cylinder (side $0.5 \lambda$, center $(0.25 \lambda,-0.5 \lambda)$, relative dielectric permittivity 1.5$)$. The cost function (6), in its multiview version, is employed. The parameters of the ABC have been set equal to as follows: $C=50, P=25$, $K_{\lim }=100$, and $k_{\max }=2000$.

Some examples of the behavior of the cost function versus the iteration number are shown in Figure 4, which reports five different runs of the algorithms. As can be seen, in all cases, the method converges to a value of about 0.15 . The corresponding mean relative reconstruction error is $3 \%$. The same configuration has been considered in [76] and solved by using an ACO-based inversion approach. In the results provided in that paper, a mean relative error of about $4.5 \%$ is achieved with the ACO-based approach. Finally, an example of the reconstructed distribution of the dielectric properties obtained by the ABC approach is shown in Figure 5. As can be seen, the two objects are correctly shaped, and their permittivity is identified with quite good accuracy.

\section{Conclusions}

In this paper, the application of swarm intelligence algorithms, that is, stochastic algorithms inspired by the collective social behavior of agents (e.g., birds, ants, etc.), to the solution of microwave imaging problem has been reviewed. 


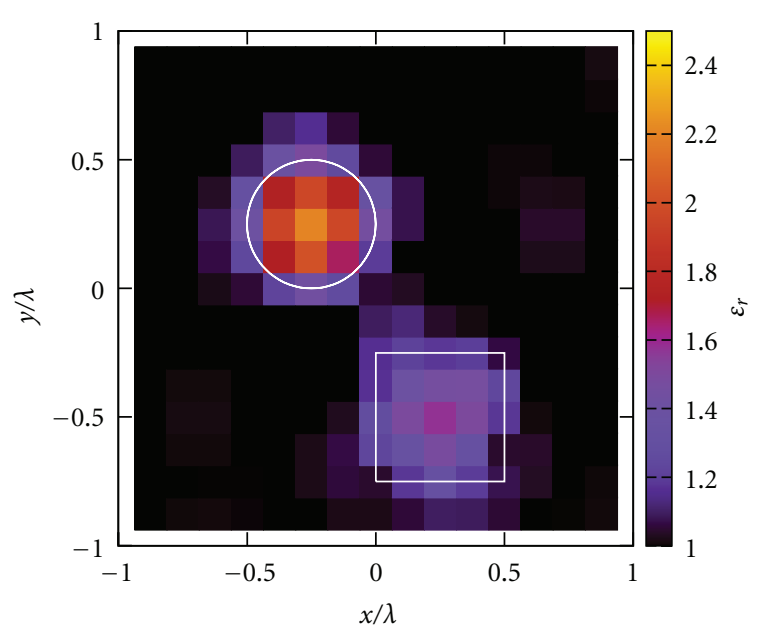

FIgURE 5: Example of reconstructed distribution of the dielectric permittivity. ABC-based inversion algorithm.

Such approaches have been proven to be very effective in several applications. The results available in the literature confirm the suitability of this class of optimization methods for microwave imaging, too.

\section{References}

[1] M. Pastorino, Microwave Imaging, John Wiley, Hoboken, NJ, USA, 2010.

[2] Y. J. Kim, L. Jofre, F. De Flaviis, and M. Q. Feng, "Microwave reflection tomographic array for damage detection of civil structures," IEEE Transactions on Antennas and Propagation, vol. 51, no. 11, pp. 3022-3032, 2003.

[3] M. Benedetti, M. Donelli, G. Franceschini, M. Pastorino, and A. Massa, "Effective exploitation of the a priori information through a microwave imaging procedure based on the SMW for NDE/NDT applications," IEEE Transactions on Geoscience and Remote Sensing, vol. 43, no. 11, pp. 2584-2591, 2005.

[4] L. Chommeloux, C. Pichot, and J. C. Bolomey, "Electromagnetic modeling for microwave imaging of cylindrical buried inhomogeneities," IEEE Transactions on Microwave Theory and Techniques, vol. 34, no. 10, pp. 1064-1076, 1986.

[5] M. Pastorino, S. Caorsi, and A. Massa, "Numerical assessment concerning a focused microwave diagnostic method for medical applications," IEEE Transactions on Microwave Theory and Techniques, vol. 48, no. 1, pp. 1815-1830, 2000.

[6] G. Bozza, C. Estatico, M. Pastorino, and A. Randazzo, "An inexact Newton method for microwave reconstruction of strong scatterers," IEEE Antennas and Wireless Propagation Letters, vol. 5, no. 1, pp. 61-64, 2006.

[7] A. G. Tijhuis, K. Belkebir, A. C. S. Litman, and B. P. De Hon, "Theoretical and computational aspects of 2-D inverse profiling," IEEE Transactions on Geoscience and Remote Sensing, vol. 39, no. 6, pp. 1316-1330, 2001.

[8] A. G. Tijhuis, K. Belkebir, A. C. S. Litman, and B. P. De Hon, "Multiple-frequency distorted-wave Born approach to 2D inverse profiling," Inverse Problems, vol. 17, no. 6, pp. 1635$1644,2001$.

[9] G. Bozza, C. Estatico, A. Massa, M. Pastorino, and A. Randazzo, "Short-range image-based method for the inspection of strong scatterers using microwaves," IEEE Transactions on
Instrumentation and Measurement, vol. 56, no. 4, pp. 11811188, 2007.

[10] G. Bozza, C. Estatico, M. Pastorino, and A. Randazzo, "Application of an inexact-Newton method within the second-order Born approximation to buried objects," IEEE Geoscience and Remote Sensing Letters, vol. 4, no. 1, pp. 51-55, 2007.

[11] C. Estatico, G. Bozza, A. Massa, M. Pastorino, and A. Randazzo, "A two-step iterative inexact-Newton method for electromagnetic imaging of dielectric structures from real data," Inverse Problems, vol. 21, no. 6, pp. S81-S94, 2005.

[12] Z. Q. Zhang and Q. H. Liu, "Three-dimensional nonlinear image reconstruction for microwave biomedical imaging," IEEE Transactions on Biomedical Engineering, vol. 51, no. 3, pp. 544-548, 2004.

[13] P. Mojabi and J. LoVetri, "Overview and classification of some regularization techniques for the Gauss-Newton inversion method applied to inverse scattering problems," IEEE Transactions on Antennas and Propagation, vol. 57, no. 9, pp. 26582665, 2009.

[14] A. Litman, D. Lesselier, and F. Santosa, "Reconstruction of a two-dimensional binary obstacle by controlled evolution of a level-set," Inverse Problems, vol. 14, no. 3, pp. 685-706, 1998.

[15] R. Autieri, G. Ferraiuolo, and V. Pascazio, "Bayesian regularization in nonlinear imaging: reconstructions from experimental data in nonlinearized microwave tomography," IEEE Transactions on Geoscience and Remote Sensing, vol. 49, no. 2, pp. 801-813, 2011.

[16] C. Gilmore, P. Mojabi, and J. LoVetri, "Comparison of an enhanced distorted born iterative method and the multiplicative-regularized contrast source inversion method," IEEE Transactions on Antennas and Propagation, vol. 57, no. 8, pp. 2341-2351, 2009.

[17] M. El-Shenawee, O. Dorn, and M. Moscoso, "An adjoint-field technique for shape reconstruction of 3-D penetrable object immersed in lossy medium," IEEE Transactions on Antennas and Propagation, vol. 57, no. 2, pp. 520-534, 2009.

[18] P. Lobel, L. Blanc-Féraud, C. Pichet, and M. Barlaud, "A new regularization scheme for inverse scattering," Inverse Problems, vol. 13, no. 2, pp. 403-410, 1997.

[19] T. M. Habashy and A. Abubakar, "A general framework for constraint minimization for the inversion of electromagnetic measurements," Progress In Electromagnetics Research, vol. 46, pp. 265-312, 2004.

[20] P. M. Van Den Berg and A. Abubakar, "Contrast source inversion method: state of art," Journal of Electromagnetic Waves and Applications, vol. 15, no. 11, pp. 1503-1505, 2001.

[21] A. Randazzo, G. Oliveri, A. Massa, and M. Pastorino, "Electromagnetic inversion with the multiscaling inexact Newton method-experimental validation," Microwave and Optical Technology Letters, vol. 53, no. 12, pp. 2834-2838, 2011.

[22] S. Caorsi, A. Costa, and M. Pastorino, "Microwave imaging within the second-order born approximation: stochastic optimization by a genetic algorithm," IEEE Transactions on Antennas and Propagation, vol. 49, no. 1, pp. 22-31, 2001.

[23] C.-C. Chiu and P. T. Liu, "Image reconstruction of a perfectly conducting cylinder by the genetic algorithm," IEE Proceedings-Microwaves, Antennas and Propagation, vol. 143, no. 3, p. 249, 1996.

[24] A. Qing, C. K. Lee, and L. Jen, "Electromagnetic inverse scattering of two-dimensional perfectly conducting objects by real-coded genetic algorithm," IEEE Transactions on Geoscience and Remote Sensing, vol. 39, no. 3, pp. 665-676, 2001.

[25] S. Caorsi, A. Massa, M. Pastorino, M. Raffetto, and A. Randazzo, "Detection of buried inhomogeneous elliptic cylinders 
by a memetic algorithm," IEEE Transactions on Antennas and Propagation, vol. 51, no. 10, pp. 2878-2884, 2003.

[26] M. Pastorino, S. Caorsi, A. Massa, and A. Randazzo, "Reconstruction algorithms for electromagnetic imaging," IEEE Transactions on Instrumentation and Measurement, vol. 53, no. 3, pp. 692-699, 2004.

[27] L. Garnero, A. Franchois, J. P. Hugonin, C. Pichot, and N. Joachimowicz, "Microwave imaging-complex permittivity reconstruction by simulated annealing," IEEE Transactions on Microwave Theory and Techniques, vol. 39, no. 11, pp. 18011807, 1991.

[28] M. Pastorino, "Stochastic optimization methods applied to microwave imaging: a review," IEEE Transactions on Antennas and Propagation, vol. 55, no. 3, pp. 538-548, 2007.

[29] Y. Rahmat-Samii and E. Michielssen, Electromagnetic Optimization by Genetic Algorithms, John Wiley \& Sons, New York, NY, USA, 1999.

[30] J. M. Johnson and Y. Rahmat-Samii, "Genetic algorithms in engineering electromagnetics," IEEE Antennas and Propagation Magazine, vol. 39, no. 4, pp. 7-21, 1997.

[31] R. L. Haupt, "Introduction to genetic algorithms for electromagnetics," IEEE Antennas and Propagation Magazine, vol. 37, no. 2, pp. 7-15, 1995.

[32] A. Massa, M. Pastorino, and A. Randazzo, "Reconstruction of two-dimensional buried objects by a differential evolution method," Inverse Problems, vol. 20, no. 6, pp. S135-S150, 2004.

[33] D. S. Weile and E. Michielssen, "genetic algorithm optimization applied to electromagnetics: a review," IEEE Transactions on Antennas and Propagation, vol. 45, no. 3, pp. 343-353, 1997.

[34] D. E. Goldberg, Genetic Algorithms in Search, Optimization, and Machine Learning, Addison-Wesley Professional, Reading, Mass, USA, 1st edition, 1989.

[35] K. V. Price, "An introduction to differential evolution," in New Ideas in Optimization, pp. 79-108, McGraw-Hill, Maidenhead, UK, 1999.

[36] E. Bonabeau, M. Dorigo, and G. Theraulaz, Swarm Intelligence?: From Natural to Artificial Intelligence, Oxford University Press, New York, NY, USA, 1999.

[37] C. Blum and D. Merkle, Swarm Intelligence Introduction and Applications, Springer, London, UK, 2008.

[38] J. F. Kennedy, R. C. Eberhart, and Y. Shi, Swarm Intelligence, Morgan Kaufmann Publishers, San Francisco, Calif, USA, 2001.

[39] J. Robinson and Y. Rahmat-Samii, "Particle swarm optimization in electromagnetics," IEEE Transactions on Antennas and Propagation, vol. 52, no. 2, pp. 397-407, 2004.

[40] M. Dorigo, V. Maniezzo, and A. Colorni, "Ant system: optimization by a colony of cooperating agents," IEEE Transactions on Systems, Man, and Cybernetics B, vol. 26, no. 1, pp. 29-41, 1996.

[41] M. Dorigo and L. M. Gambardella, "Ant colony system: a cooperative learning approach to the traveling salesman problem," IEEE Transactions on Evolutionary Computation, vol. 1, no. 1 , pp. 53-66, 1997.

[42] C. Estatico, M. Pastorino, and A. Randazzo, "A novel microwave imaging approach based on regularization in Lp Banach spaces," IEEE Transactions on Antennas and Propagation, vol. 60, no. 7, pp. 3373-3381, 2012.

[43] G. Bilchev and I. C. Parmee, "The ant colony metaphor for searching continuous design spaces," in Proceedings of the Selected Papers from AISB Workshop on Evolutionary Computing, pp. 25-39, Sheffield, UK, 1995.

[44] J. Dréo and P. Siarry, "A new ant colony algorithm using the heterarchical concept aimed at optimization of multiminima continuous functions," in Proceedings of the 3rd International Workshop on Ant Algorithms (ANTS '02), pp. 216-221, London, UK, 2002.

[45] N. Monmarché, G. Venturini, and M. Slimane, "On how Pachycondyla apicalis ants suggest a new search algorithm," Future Generation Computer Systems, vol. 16, no. 8, pp. 937$946,2000$.

[46] K. Socha and M. Dorigo, "Ant colony optimization for continuous domains," European Journal of Operational Research, vol. 185, no. 3, pp. 1155-1173, 2008.

[47] D. Karaboga, "An idea based on honey bee swarm for numerical optimization," Tech. Rep. TR06, Erciyes University, Kayseri, Turkey, 2005.

[48] D. Karaboga and B. Basturk, "Artificial Bee Colony (ABC) optimization algorithm for solving constrained optimization problems," in Foundations of Fuzzy Logic and Soft Computing, P. Melin, O. Castillo, L. T. Aguilar, J. Kacprzyk, and W. Pedrycz, Eds., vol. 4529, pp. 789-798, Springer, Berlin, Germany.

[49] V. Tereshko and A. Loengarov, "Collective decision making in honey-bee foraging dynamics," Computing and Information Systems, vol. 9, no. 3, pp. 1-7, 2005.

[50] A. Semnani, M. Kamyab, and I. T. Rekanos, "Reconstruction of one-dimensional dielectric scatterers using differential evolution and particle swarm optimization," IEEE Geoscience and Remote Sensing Letters, vol. 6, no. 4, pp. 671-675, 2009.

[51] A. Semnani and M. Kamyab, "Comparison of differential evolution and particle swarm optimization in one-dimensional reconstruction problems," in Proceedings of the Asia Pacific Microwave Conference (APMC '08), pp. 1-4, Hong Kong, December 2008

[52] A. M. Emad Eldin, E. A. Hashish, and M. I. Hassan, "Inversion of lossy dielectric profiles using particle swarm optimization," Progress In Electromagnetics Research M, vol. 9, pp. 93-105, 2009.

[53] A. Fhager, A. Voronov, C. Chen, and M. Persson, "Methods for dielectric reconstruction in microwave tomography," in Proceedings of the 2nd European Conference on Antennas and Propagation (EuCAP '07), pp. 1-6, Edinburgh, UK, November 2007.

[54] C.-H. Huang, C. C. Chiu, C. L. Li, and K. C. Chen, "Time domain inverse scattering of a two-dimensional homogenous dielectric object with arbitrary shape by particle swarm optimization," Progress in Electromagnetics Research, vol. 82, pp. 381-400, 2008.

[55] S. Caorsi, M. Donelli, A. Lommi, and A. Massa, "Location and imaging of two-dimensional scatterers by using a particle swarm algorithm," Journal of Electromagnetic Waves and Applications, vol. 18, no. 4, pp. 481-494, 2004.

[56] H. Zhang, X. D. Zhang, J. Ji, and Y. J. Yao, "Electromagnetic imaging of the 2-D media based on Particle Swarm algorithm," in Proceedings of the 6th International Conference on Natural Computation (ICNC '10), pp. 262-265, Yantai, China, August 2010.

[57] S. H. Zainud-Deen, W. M. Hassen, and K. H. Awadalla, "Techniques, crack detection using a hybrid finite difference frequency domain and particle swarm optimization," in Proceedings of the National Radio Science Conference (NRSC '09), pp. 1-8, Cairo, Egypt, March 2009.

[58] S. H. Zainud-Deen, W. M. Hassen, E. M. Ali, K. H. Awadalla, and H. A. Sharshar, "Breast cancer detection using a hybrid finite difference frequency domain and particle swarm optimization techniques," in Proceedings of the 25th National Radio Science Conference (NRSC '08), pp. 1-8, Tanta, Egypt, March 2008. 
[59] I. T. Rekanos and M. Kanaki, "Microwave imaging of twodimensional conducting scatterers using particle swarm optimization," in Electromagnetic Fields in Mechatronics, Electrical and Electronic Engineering, A. Krawczyk, S. Wiak, and L. M. Fernandez, Eds., pp. 84-89, IOS Press, Amsterdam, The Netherlands, 2006.

[60] I. T. Rekanos, "Shape reconstruction of a perfectly conducting scatterer using differential evolution and particle swarm optimization," IEEE Transactions on Geoscience and Remote Sensing, vol. 46, no. 7, pp. 1967-1974, 2008.

[61] T. Huang and A. S. Mohan, "Application of particle swarm optimization for microwave imaging of lossy dielectric objects," in Proceedings of the IEEE Antennas and Propagation Society International Symposium and USNC/URSI Meeting, vol. 1B, pp. 852-855, Washington, DC, USA, July 2005.

[62] T. Huang and A. Sanagavarapu Mohan, "A microparticle swarm optimizer for the reconstruction of microwave images," IEEE Transactions on Antennas and Propagation, vol. 55, no. 3 , pp. 568-576, 2007.

[63] C.-L. Li, C. C. Chiu, and C. H. Huang, "Time domain inverse scattering for a homogenous dielectric cylinder by asynchronous particle swarm optimization," Journal of Testing and Evaluation, vol. 39, no. 3, 2011.

[64] C. Wei, C. H. Sun, J. O. Wu, C. C. Chiub, and M. K. Wu, "Inverse scattering for the perfectly conducting cylinder by asynchronous particle swarm optimization," in Proceedings of the 3rd IEEE International Conference on Communication Software and Networks, pp. 410-4412, Xi' an, China, 2011.

[65] C.-H. Sun, C. C. Chiu, and C. L. Li, "Time-domain inverse scattering of a two-dimensional metallic cylinder in slab medium using asynchronous particle swarm optimization," Progress In Electromagnetics Research M, vol. 14, pp. 85-100, 2010.

[66] B. Mhamdi, K. Grayaa, and T. Aguili, "Microwave imaging for conducting scatterers by hybrid particle swarm optimization with simulated annealing," in Proceedings of the 8th International Multi-Conference on Systems, Signals and Devices (SSD '11), pp. 1-6, Sousse, Tunisia, March 2011.

[67] G. R. Huang, W. J. Zhong, and H. W. Liu, "Microwave imaging based on the AWE and HPSO incorporated with the information obtained from born approximation," in Proceedings of the International Conference on Computational Intelligence and Software Engineering (CiSE '10), pp. 1-4, Wuhan, Chin, December 2010.

[68] B. Mhamdi, K. Grayaa, and T. Aguili, "An inverse scattering approach using hybrid PSO-RBF network for microwave imaging purposes," in Proceedings of the 16th IEEE International Conference on Electronics, Circuits and Systems (ICECS '09), pp. 231-234, Hammamet, Tunisia, December 2009.

[69] M. Donelli, G. Franceschini, A. Martini, and A. Massa, "An integrated multiscaling strategy based on a particle swarm algorithm for inverse scattering problems," IEEE Transactions on Geoscience and Remote Sensing, vol. 44, no. 2, pp. 298-312, 2006.

[70] M. Donelli and A. Massa, "Computational approach based on a particle swarm optimizer for microwave imaging of twodimensional dielectric scatterers," IEEE Transactions on Microwave Theory and Techniques, vol. 53, no. 5, pp. 1761-1776, 2005.

[71] D. Franceschini and A. Massa, "An integrated stochastic multi-scaling strategy for microwave imaging applications," in Proceedings of the IEEE Antennas and Propagation Society International Symposium and USNC/URSI Meeting, pp. 209-212, Washington, DC, USA, July 2005.
[72] G. Franceschini, M. Donelli, R. Azaro, and A. Massa, "Inversion of phaseless total field data using a two-step strategy based on the iterative multiscaling approach," IEEE Transactions on Geoscience and Remote Sensing, vol. 44, no. 12, pp. 3527-3539, 2006.

[73] R. Azaro, D. Franceschini, G. Franceschini, L. Manica, and A. Massa, "A two-step strategy for a multi-scaling inversion of phaseless measurements of the total field," in Proceedings of the IEEE Antennas and Propagation Society International Symposium, APS 2006, pp. 1073-1076, Albuquerque, NM, USA, July 2006.

[74] M. Donelli, D. Franceschini, P. Rocca, and A. Massa, "Threedimensional microwave imaging problems solved through an efficient multiscaling particle swarm optimization," IEEE Transactions on Geoscience and Remote Sensing, vol. 47, no. 5, pp. 1467-1481, 2009.

[75] D. Franceschini, G. Franceschini, M. Donelli, P. Rocca, and A. Massa, "Imaging three-dimensional bodies by processing multi-frequency data through a multiscale swarm intelligence based method," in Proceedings of the IEEE Antennas and Propagation Society International Symposium (AP-S '07), pp. 409412, Honolulu, Hawaii, USA, June 2007.

[76] M. Pastorino and A. Randazzo, "Nondestructive analysis of dielectric bodies by means of an Ant Colony Optimization method," in Swarm Intelligence for Electric and Electronic Engineering, G. Fornarelli and L. Mescia, Eds., IGI Global, Hershey, $\mathrm{Pa}, \mathrm{USA}, 2012$.

[77] M. Brignone, G. Bozza, A. Randazzo, R. Aramini, M. Piana, and M. Pastorino, "Hybrid approach to the inverse scattering problem by using ant colony optimization and no-sampling linear sampling," in Proceedings of the IEEE International Symposium on Antennas and Propagation and USNC/URSI National Radio Science Meeting (APSURSI '08), pp. 1-4, San Diego, Calif, USA, July 2008.

[78] G. Bozza, M. Brignone, M. Pastorino, M. Piana, and A. Randazzov, "An inverse scattering based hybrid method for the measurement of the complex dielectric permittivities of arbitrarily shaped homogenous targets," in Proceedings of the IEEE Intrumentation and Measurement Technology Conference (I2MTC '09), pp. 719-723, Singapore, May 2009.

[79] M. Brignone, G. Bozza, A. Randazzo, M. Piana, and M. Pastorino, "A hybrid approach to $3 \mathrm{D}$ microwave imaging by using linear sampling and ACO," IEEE Transactions on Antennas and Propagation, vol. 56, no. 10, pp. 3224-3232, 2008.

[80] M. Donelli, I. Craddock, D. Gibbins, and M. Sarafianou, "A three-dimensional time domain microwave imaging method for breast cancer detection based on an evolutionary algorithm," Progress In Electromagnetics Research M, vol. 18, pp. 179-195, 2011.

[81] K. Belkebir and M. Saillard, "Special section: testing inversion algorithms against experimental data," Inverse Problems, vol. 17, no. 6, p. 1565, 2001.

[82] K. Tenglong, Z. Xiaoying, W. Jian, and D. Yihan, "A modified ACO algorithm for the optimization of antenna layout," in Proceedings of the 2011 International Conference on Electrical and Control Engineering, pp. 4269-4272, Yichang, China, 2011.

[83] G. Weis, A. Lewis, M. Randall, and D. Thiel, "Pheromone preseeding for the construction of RFID antenna structures using ACO," in Proceedings of the 6th IEEE International Conference on e-Science, eScience 2010, pp. 161-167, Brisbane, Australia, December 2010. 
[84] I. Vilovic, N. Burum, Z. Sipus, and R. Nad, "PSO and ACO algorithms applied to location optimization of the WLAN base station," in Proceedings of the 19th International Conference on Applied Electromagnetics and Communications (ICECom '07), pp. 1-5, Dubrovnik, Croatia, September 2007.

[85] R. Harrington, Field Computation by Moment Methods, IEEE Press, Piscataway, NJ, USA, 1993. 

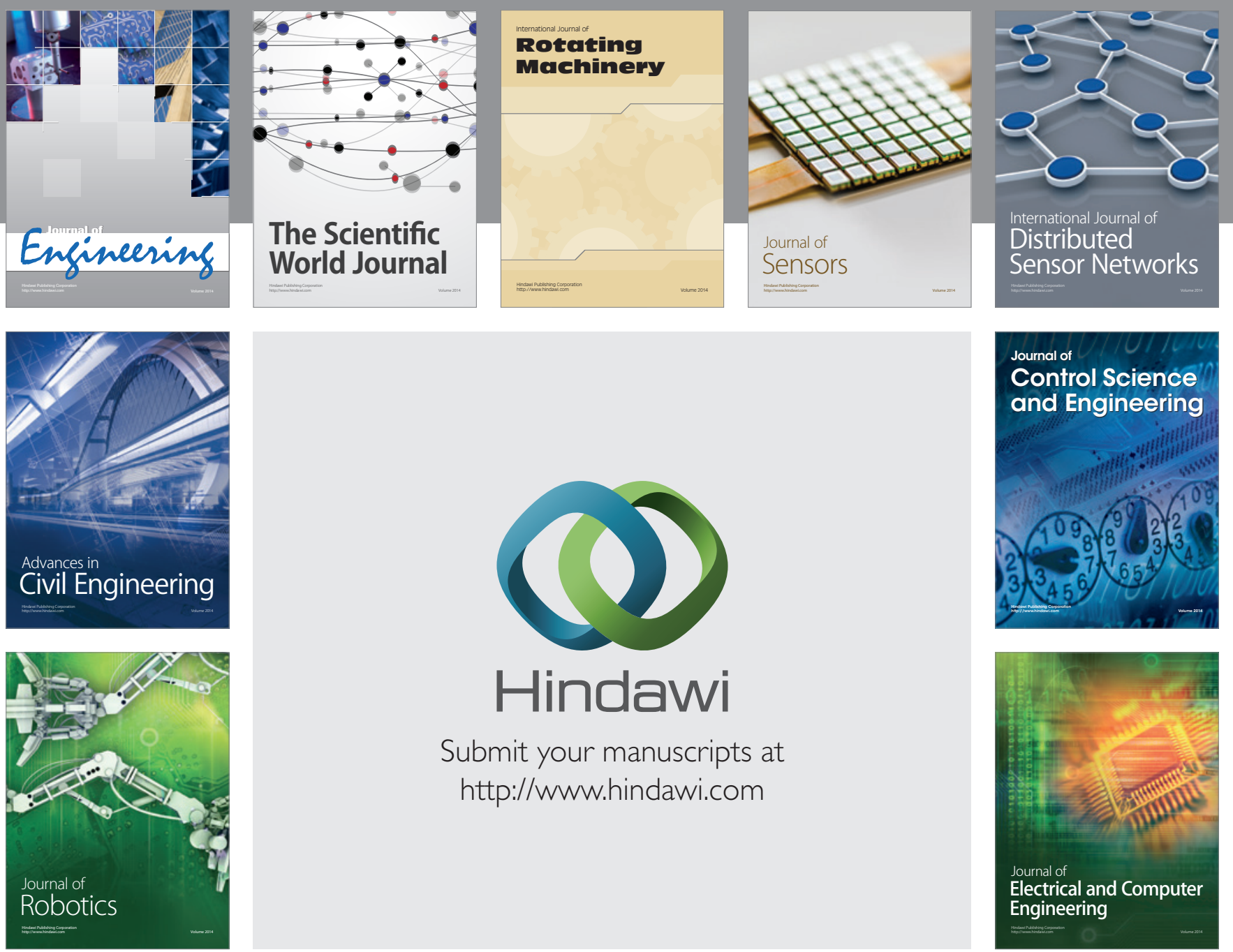

Submit your manuscripts at

http://www.hindawi.com
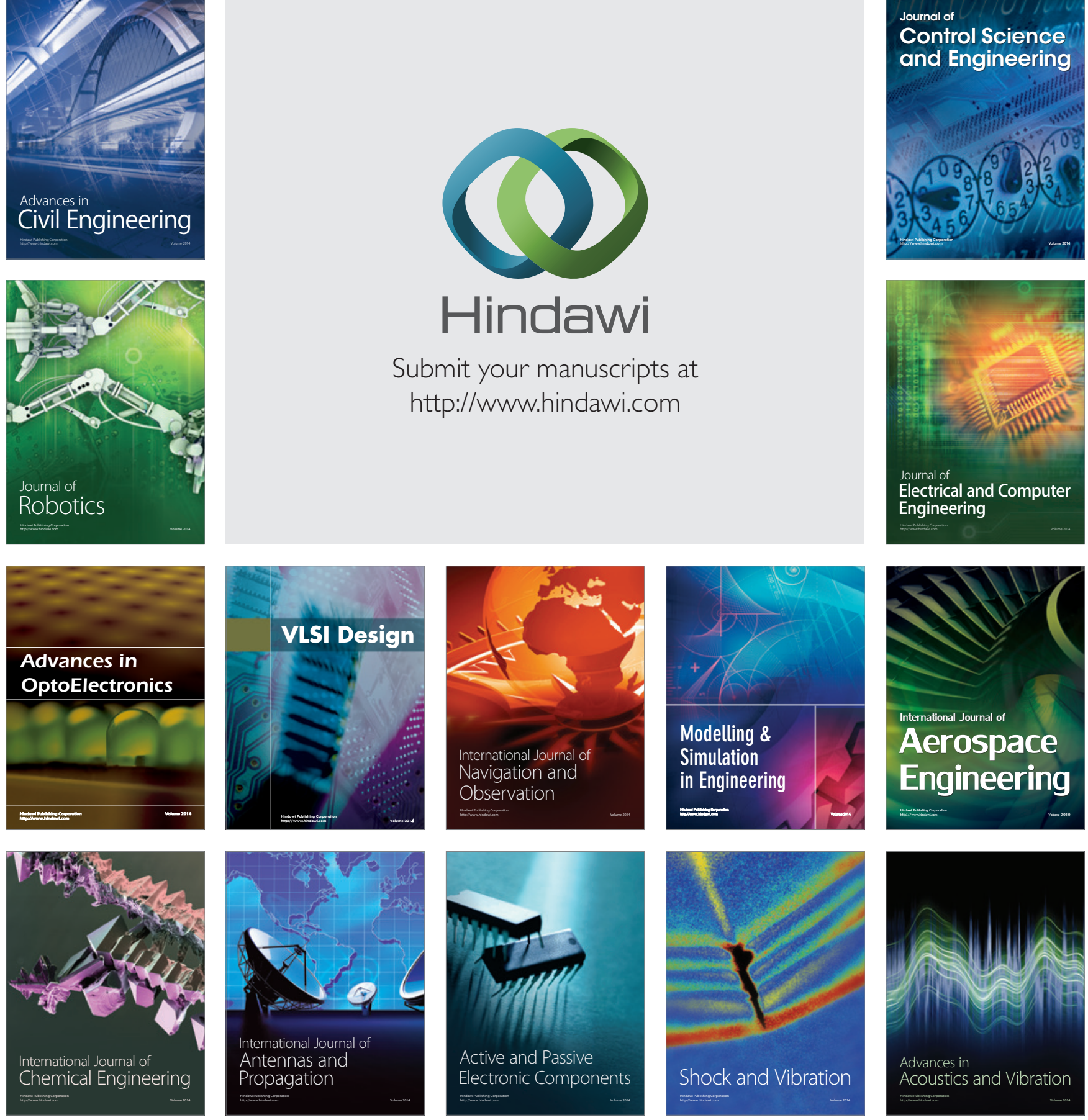